\title{
Anti-BCMA Antibody SEA-BCMA
}

National Cancer Institute

\section{Source}

National Cancer Institute. Anti-BCMA Antibody SEA-BCMA. NCI Thesaurus. Code C148176.

A humanized, afucosylated monoclonal antibody created using the proprietary, sugarengineered antibody (SEA) platform and directed against the tumor-associated antigen (TAA) human B-cell maturation antigen (BCMA; TNFRSF17), with potential immunoadjuvant activity. Upon administration, the anti-BCMA antibody SEA-BCMA targets and binds to BCMA expressed on tumor cells. When administered with antibodycoupled T-cell receptor (ACTR)-expressing T-cells, the ACT R-expressing T-cells bind, with high affinity, to the anti-BCMA antibody SEA-BCMA. This activates the ACT R T-cells and the T-cells induce specific cytotoxic T-lymphocyte (CTL)-mediated cytotoxicity toward BCMA-expressing tumor cells. BCMA, a cell surface protein and member of the tumor necrosis factor (TNF) receptor superfamily that is specifically overexpressed on malignant plasma cells, plays a key role in promoting plasma cell survival. 